\title{
The Value of Carlsson-Dent Questionnaire in Diagnosis of Gastroesophageal Reflux Disease in Area With Low Prevalence of Gastroesophageal Reflux Disease
}

Nisa Netinatsunton, Siriboon Attasaranya, Bancha Ovartlarnporn, ${ }^{*}$ Sulee Sangnil, Sopa Boonviriya and Teerha Piratvisuth

NKC Institute of Gastroenterology and Hepatology, Songklanagarind Hospital, Faculty of Medicine, Prince of Songkla University, Hatyai,

Songkhla, Thailand

\section{Background/Aims}

Symptom-based diagnosis for gastroesophageal reflux disease (GERD) has been accepted in the population with high prevalence. Carlsson-Dent questionnaire (CDQ) is a standardized symptom-based diagnosis tool for GERD. The value of this tool in the population with low prevalence is unknown. The aim of this study was to determine CDQ performance for diagnosis of GERD in Thai population with low prevalence versus endoscopy or 24 hour pH monitoring.

\section{Methods}

Patients with dyspepsia by Rome II criteria were recruited. All patients completed a Thai version of CDQ and underwent endoscopic examination. Those without esophagitis or peptic ulcer and positive CDQ score took pH monitoring.

Results

One hundred patients (68 female) with mean age \pm SD of $45.6 \pm 12.4$ years were recruited. Six with Los Angeles grade $A$ esophagitis had negative CDQ score. In 44 with positive CDQ score, 3 had Los Angeles grade B esophagitis and 41 had pH monitoring done with 8 having positive test. The GERD diagnosis by CDQ was confirmed in 11 of 44 patients (25\%). CDQ detected 11 out of 17 GERD detected by endoscopy and pH monitoring and the sensitivity of CDQ was 64\%.

\section{Conclusions}

CDQ diagnosed more GERD in Thai population with low prevalence compared with endoscopy and pH monitoring. This may be due to some patients with functional heartburn were picked up by CDQ and some patients with GERD were not detected by endoscopy and $\mathrm{pH}$ monitoring.

(J Neurogastroenterol Motil 2011;17:164-168)

\section{Key Words}

Diagnosis; Gastroesophageal Reflux; Questionnaires

Received: January 5, 2011 Revised: February 6, 2011 Accepted: February 28, 2011

(c) This is an Open Access article distributed under the terms of the Creative Commons Attribution Non-Commercial License (http://creativecommons. org/licenses/by-nc/3.0) which permits unrestricted non-commercial use, distribution, and reproduction in any medium, provided the original work is properly cited.

*Correspondence: Bancha Ovartlarnporn, MD

NKC Institute of Gastroenterology and Hepatology, 8th Floor Chalermprabaramee Buiding, Songklanagarind Hospital, Faculty of Medicine, Prince of Songkla University, Hatyai, Songkhla 90110, Thailand

Financial support: None.

Tel: +66-74-451965-9, Fax: +66-74-429436, E-mail: mrovart@ji-net.com

Conflicts of interest: None. 


\section{Introduction}

Gastroesophageal reflux disease (GERD) is a common problem in general practice. The reported prevalence of GERD using different definitions varied from 10\%-30\% in the Western population. ${ }^{1-3}$ The prevalence of GERD in Eastern Asia ranged from $2.5 \%-6.7 \%$ and 1 cross-sectional study from Singapore showed the prevalence of $10.5 \% .^{4-6}$ No published data regarding the prevalence of GERD in Thailand are available. The study of Thai motility club using questionnaire reported $7.4 \%$ prevalence of GERD in community (unpublished data). The prevalence of esophagitis as detected by endoscopy from data collected by the stomach research group of Thailand during the process of developing dyspepsia guideline 10 years ago ranged from 4\%-6\% (unpublished data). We recently analyzed our prospectively stored computerized endoscopic database in 1,320 patients with dyspepsia between the year 2007-2008 and the prevalence of esophagitis was $4 \%$ (unpublished data). Based on these data, the prevalence of GERD in Thailand seems to be the same as other East Asian countries.

Heartburn and/or acid regurgitation are regarded as typical symptoms for GERD. Symptoms based-diagnosis is widely utilized in the western countries with acceptable sensitivity and specificity. ${ }^{7}$ Some structural questionnaires were developed to standardize the symptom- based diagnosis of GERD. ${ }^{8-15}$ The studies evaluating the validity of these types of questionnaire have conflicting results with reported sensitivity of $60 \%-92 \%$. $^{9-18}$ Carlsson and colleagues developed a self-administered questionnaire that focused on the nature of the sensations experienced by the patient as well as provoking, exacerbating and relieving factors. The questionnaire had a sensitivity of $92 \%$ and specificity of $19 \%$ in diagnosis of GERD compared with endoscopic esophagitis and 24 hour $\mathrm{pH}$ monitoring as standard. ${ }^{13}$ The prevalence of GERD in the target population could influence the performance of symptom-based diagnosis of GERD. ${ }^{5,7}$ The clinical value of this tool in the population with low prevalence of GERD is unknown. Thus, the aim of this study is to assess the usefulness of the Carlsson-Dent questionnaire (CDQ) in diagnosis of GERD in the population with low prevalence of GERD.

\section{Materials and Methods}

\section{Patients}

One hundred patients with dyspeptic symptoms by Rome II criteria with or without gastroesophageal reflux symptoms and age more than 18 years scheduled for endoscopy were prospectively enrolled from May 2007 to January 2008. Patients were excluded if they had alarm symptoms, a history of documented ulcer disease, gastric surgery, gastric cancer, or severe concomitant medical conditions. An informed consent for each patient was obtained before the entry to the study.

\section{Study Site}

This study was conducted at the NKC Institute of Gastroenterology and Hepatology, Faculty of Medicine, Prince of Songkla University which is a tertiary referring center for gastroenterology in the South of Thailand.

\section{Study Protocol}

All patients completed the Thai version of CDQ that had been validated by back translation. CDQ score of equal to or greater than 4 is a positive score for GERD. The baseline characteristics (age, gender, body weight, height, smoking history and drinking history), predominant symptoms and duration of symptoms before endoscopy were obtained.

Endoscopic examination was done in standard manner after patient had completed CDQ form. The endoscopists were not aware of patients' CDQ score. The endoscopic diagnosis of esophagitis and grading were based on Los Angeles (LA) classification. ${ }^{19}$ Barrett's esophagus was defined as the presence of specialized columnar epithelium equal or more than three centimeters above the gastric fold with confirmed histological study. ${ }^{20}$ The significant findings were defined as gastric ulcer, gastric cancer, duodenal ulcer, severe erosive gastritis and severe erosive duodenitis.

Patients with erosive esophagitis, peptic ulcer, severe erosive gastritis, severe erosive duodenitis or score of CDQ less than 4 did not proceed to have 24 hour $\mathrm{pH}$ monitoring done. Those with negative or insignificant findings from endoscopy and score of CDQ equal or greater than four were scheduled for 24 hour pH monitoring study (Figure).

The ambulatory 24 hour esophageal $\mathrm{pH}$ monitoring was done with a $\mathrm{pH}$ probe placed 6 centimeters above the proximal 


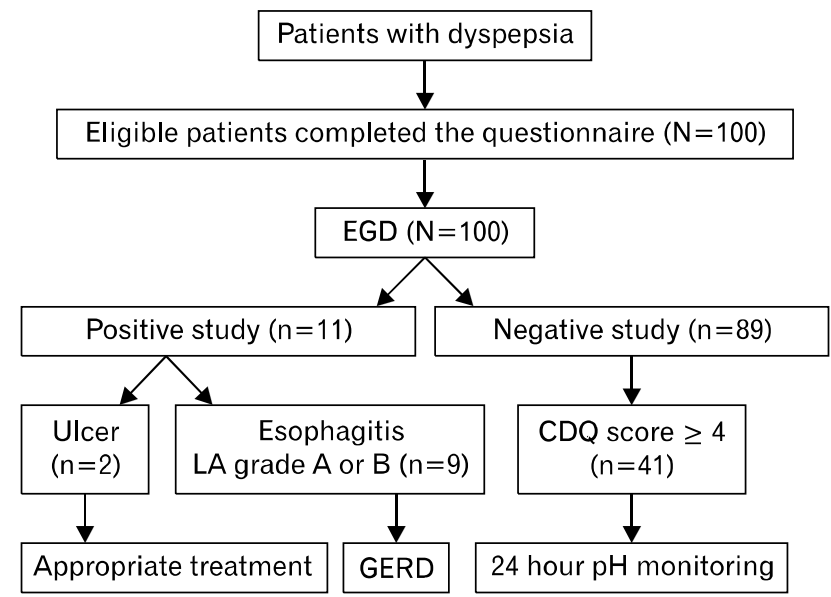

Figure. The flow diagram of the study. EGD, Esophago-gastroduodenal endoscopy; LA, Los Angeles; CDQ, Carlsson-Dent questionnaire; GERD, gastroesophageal reflux disease.

margin of Z-line as measured during endoscopy while withdrawing the gastroscope using Digitrapper ${ }^{\mathbb{R}} \mathrm{pH} 400$ (Medtronic, Skovlunde, Denmark). Proton Pump inhibitor (PPI) was stopped for at least 1 week before the study. Subjects were provided a diary to record meal times, position changes and the time and type of symptoms encountered during the recording period. They were specifically instructed to avoid acidic fluid such as citrus fruit juice or any condiment containing vinegar, to pursue their daily activities and to have their usual diet. Data of 24 hour $\mathrm{pH}$ study were analyzed by a computer software (Medtronic). A positive 24 hour $\mathrm{pH}$ monitoring study was defined by the percentage of total time of $\mathrm{pH}$ below 4 being more than $5 \% .{ }^{21}$ Patients with a positive test were diagnosed as having GERD.

\section{Sample Size}

Since there is no data regarding the performance of $\mathrm{CDQ}$ in low GERD prevalence population, 100 patients were arbitrarily selected to explore our concept.

The study protocol has been approved by the ethic committee of the faculty of medicine, Prince of Songkla University.

\section{Statistical Methods}

Data were analyzed by descriptive statistics and were expressed as mean with standard deviation and as frequency with percentage where appropriate.
Table 1. The Predominant Symptoms, Duration of Symptoms and Endoscopic Findings in 100 Patients

\begin{tabular}{|c|c|}
\hline & $\begin{array}{c}\text { Number of } \\
\text { patients }\end{array}$ \\
\hline \multicolumn{2}{|l|}{ Predominant symptom } \\
\hline Heartburn & 37 \\
\hline Regurgitation & 4 \\
\hline Upper abdominal pain & 25 \\
\hline Nausea/vomiting & 9 \\
\hline Other symptoms ${ }^{a}$ & 25 \\
\hline Total & 100 \\
\hline \multicolumn{2}{|c|}{ Duration of symptoms prior to endoscopy } \\
\hline$<1 \mathrm{mo}$ & 8 \\
\hline$>1 \mathrm{mo}$ to $1 \mathrm{yr}$ & 50 \\
\hline$>1 \mathrm{yr}$ & 42 \\
\hline Total & 100 \\
\hline \multicolumn{2}{|l|}{ Endoscopic finding } \\
\hline \multicolumn{2}{|l|}{ Reflux esophagitis } \\
\hline Grade A & 6 \\
\hline Grade B & 3 \\
\hline Grade C & 0 \\
\hline Grade D & 0 \\
\hline Duodenal ulcer & 1 \\
\hline Gastric ulcer & 1 \\
\hline Non-erosive gastritis & 12 \\
\hline Non-erosive duodenitis & 4 \\
\hline Hiatal hernia & 4 \\
\hline Gastric polyp & 1 \\
\hline Normal findings & 68 \\
\hline Total & 100 \\
\hline
\end{tabular}

${ }^{\mathrm{a}}$ Fullness, early satiety and discomfort.

\section{Results}

There were 100 patients recruited from May 2007 to January 2008 including 68 female and 32 male and the mean age \pm SD was $45.6 \pm 12.4$ years with a range of 25-78 years. The predominant symptoms were heartburn in 37 (37\%), upper abdominal pain in 25 (25\%), other symptoms (fullness, early satiety and discomfort) in 25 (25\%) nausea/vomiting in $9(9 \%)$ and acid regurgitation in 4 (4\%). The duration of symptoms was longer than 1 month in $92 \%$ and $42 \%$ had symptoms for more than 1 year. The endoscopic examinations were normal in 68 (68\%). Eleven patients had significant lesions, 9 with erosive esophagitis (6 of LA grade A and 3 of LA grade B), 1 with gastric ulcer and 1 with duodenal ulcer. Twenty-one patients had insignificant lesions including 12 with gastritis, 4 with duodenitis, 4 with hiatal hernia and 1 with gastric polyp. None of the patients had Barrett's 
Table 2. Carlsson-Dent Questionnaire Score, Endoscopic Esophagitis, $\mathrm{H}$ Study and Gastroesophageal Reflux Disease Diagnosis

\begin{tabular}{|c|c|c|}
\hline & \multicolumn{2}{|r|}{ CDQ score } \\
\hline & Positive & Negative \\
\hline $\begin{array}{l}\text { LA grade A or B } \\
\text { esophagitis }\end{array}$ & 3 & 6 \\
\hline All others ${ }^{\mathrm{a}}$ & 41 & 50 \\
\hline \multirow[t]{3}{*}{ Total } & 44 & 56 \\
\hline & \multicolumn{2}{|r|}{ pH study } \\
\hline & Positive & Negative \\
\hline \multirow{3}{*}{$\begin{array}{l}41 \text { with positive } \\
\text { CDQ score and negative } \\
\text { endoscopic esophagitis }\end{array}$} & & \\
\hline & 8 & 33 \\
\hline & By CDQ & $\begin{array}{l}\text { Number of cases in this group } \\
\text { that were confirmed by } \\
\text { endoscopy } / \mathrm{pH} \text { study }\end{array}$ \\
\hline \multirow[t]{3}{*}{ GERD diagnosed } & 44 & 11 \\
\hline & \multicolumn{2}{|r|}{ CDQ score } \\
\hline & Positive & Negative \\
\hline $\begin{array}{l}\text { All GERD } \\
\text { by endoscopy }+ \text { pH study }\end{array}$ & 11 & 6 \\
\hline
\end{tabular}

${ }^{a}$ Normal endoscopy, duodenal ulcer, gastric ulcer and insignificant lesions.

CDQ, Carlsson-Dent Questionnaire; LA, Los Angeles.

Data are expressed as number.

esophagus or cancer (Table 1).

Forty-four cases were diagnosed with GERD by positive CDQ score. All 3 patients with LA grade B esophagitis showed positive CDQ score whereas all 6 patients with LA grade A esophagitis showed negative CDQ score. The remaining 41 patients $(41 \%)$ with negative or insignificant endoscopic examination had 24 hour $\mathrm{pH}$ monitoring performed and 8 patients in this group presented with positive 24 hour $\mathrm{pH}$ monitoring tests. Overall, the diagnosis of GERD by CDQ was confirmed in 11 of 44 (25\%) using endoscopy and $\mathrm{pH}$ monitoring as standard and CDQ missed 6 patients with LA grade A esophagitis (Table 2). CDQ was able to identify 11 of 17 cases with GERD also detected by endoscopy and 24 hour $\mathrm{pH}$ monitoring with the sensitivity of $64 \%$.

\section{Discussion}

GERD is a common problem encountered in clinical practice. Many modalities of diagnostic tools are available for GERD diagnosis, ie, symptom-based, endoscopy, PPI test and 24 hour
$\mathrm{pH}$ monitoring. Symptom-based diagnosis is a readily available tool to all physicians. It creates neither discomfort nor complications compared to other modalities. Most of the guidelines for dyspepsia management accepted symptom-based diagnosis to exclude GERD from dyspepsia and this approach is widely practiced in the Western society with high prevalence of GERD ${ }^{22-25}$ with acceptable sensitivity and specificity. ${ }^{7}$ Structural questionnaire is one symptom-based diagnostic tool for GERD that had been developed to standardize this method. Various types of structural questionnaire had been reported in the literature ${ }^{8-15}$ and studies pertaining to the performance of these questionnaires yielded conflicting results. ${ }^{9-18}$ Currently, no structural questionnaire reported in the literature has yet met the criteria of perfect tool as proposed by Stanghellini and colleagues. ${ }^{8}$ The CDQ was reported by Carlsson et $\mathrm{al}^{13}$ to have a sensitivity of $92 \%$ and specificity of $19 \%$. Numans et $\mathrm{al}^{16}$ evaluated the CDQ in primary care setting and found that the performance of CDQ was not better than chance diagnosis by flipping a coin. Hung et $\mathrm{al}^{17}$ reported a poor performance of CDQ in predicting esophagitis and only $16 \%$ of patients with positive CDQ score had esophagitis detected by endoscopy. Factors that may influence the accuracy of symptom-based tools in GERD diagnosis include the prevalence of GERD in population studied, culture, ethnics, care setting and perception of the patients. The sensitivity of a tool will increase in proportion to the prevalence of disease in the population. ${ }^{5,7}$ The sensitivity of CDQ in our study was $64 \%$ and this may be an overestimation due to the omission of $\mathrm{pH}$ monitoring in patients with insignificant endoscopic findings with CDQ score less than four will miss some patients with GERD. Only $25 \%$ of patients with GERD diagnosed by CDQ was confirmed by endoscopy or 24 hour $\mathrm{pH}$ monitoring in our study. Our data together with data of Hung et $\mathrm{al}^{17}$ showed a poor performance of CDQ in the area with low prevalence of GERD. The diagnosis of GERD by CDQ alone may lead to inappropriate management of patients if the physicians are pressing on with the GERD treatment. Some new versions of questionnaire such as frequency scale for the symptoms of GERD, S scale and others has been studied in Asian countries with promising results. ${ }^{10-12,14,15}$ Further studies to validate these questionnaire in different population are needed to confirm the validity of these tools.

The limitations of our study include the followings: (1) the studied population was from a tertiary centre so it may not represent the population in the primary care setting where symptom-based diagnosis is the primary tool; (2) the selection bias may increase the number of patients with functional heartburn 
and this may account for, to some extent, the increased number of patients with positive CDQ score without pathological GERD; (3) the number of the patients was rather small; (4) the omission of $\mathrm{pH}$ monitoring in patient with insignificant endoscopic findings with CDQ score less than 4 might have missed some patients with GERD and the full assessment of CDQ performance in terms of sensitivity, specificity, positive predictive value and negative predictive value could not be performed; and (5) the using of $24 \mathrm{pH}$ monitoring with abnormal acid exposure time alone as a gold standard for the diagnosis of non-erosive GERD may have misclassified patients with GERD who had normal acid exposure and positive symptom sensitivity index, and patients with weak acid reflux or non-acid reflux.

In conclusion, CDQ in our study of low GERD prevalence population diagnosed more GERD than endoscopy or $\mathrm{pH}$ monitoring. This may be because some patients with functional heartburn had been picked up by CDQ and some patients with GERD were not detected by endoscopy or $\mathrm{pH}$ monitoring. Due to limited availability of other diagnostic tools in this region, it is acceptable to use symptom-based diagnosis with a reserve that it may misclassify functional heartburn as GERD and the caring physicians should be willing to refer if poor or no response to PPI treatment occur rather than pressing on adding medication aiming to treat GERD.

\section{References}

1. Dent J, El-Serag HB, Wallander MA, Johansson S. Epidemiology of gastro-oesophageal reflux disease: a systematic review. Gut 2005; 54:710-717.

2. Kang JY. Systematic review: geographical and ethnic differences in gastro-oesophageal reflux disease. Aliment Pharmacol Ther 2004; 20:705-717.

3. Fass R. Symptom assessment tools for gastroesophargeal reflux disease (GERD) treatment. J Clin Gastroenterol 2007;41:437-444.

4. Wong BC, Kinoshita Y. Systematic review on epidemiology of gastroesophageal reflux disease in Asia. Clin Gastroenterol Hepatol 2006;4:398-407.

5. Wu JC. Gastroesophageal reflux disease: an Asian perspective. J Gastroenterol Hepatol 2008;23:1785-1793.

6. Jung HK. Epidemiology of gastroesophageal reflux disease in Asia: a systematic review. J Neurogastroenterol Motil 2011;17:14-27.

7. Shaw M. Diagnostic utility of reflux disease symptoms. GUT 2004;53(suppl 4):iv25-iv27.

8. Stanghellini V, Armstrong D, Mönnikes H, Bardhan KD. Systematic review: do we need a new gastro-oesophageal reflux disease questionnaire? Aliment Pharmacol Ther 2004;19:463-479.

9. Jones R, Junghard O, Dent J, et al. Development of the GerdQ, a tool for the diagnosis and management of gastro-oesophageal reflux disease in primary care. Aliment Pharmacol Ther 2009;30:10301038.

10. Wong WM, Lam KF, Lai KC, et al. A validated symptoms questionnaire (Chinese GERDQ) for the diagnosis of gastro-oesophageal reflux disease in Chinese population. Aliment Pharmacol Ther 2003;17:1407-1413.

11. Ho KY, Gwee KA, Khor JL, Selamat DS, Yeoh KG. Validation of a graded response questionnaire for the diagnosis of gastroesophageal reflux disease in an Asian primary care population. J Clin Gastroenterol 2008;42:680-686.

12. Wang JH, Luo JY, Dong L, Gong J, Zuo AL. Composite score of reflux symptoms in diagnosis of gastroesophageal reflux disease. World J Gastroenterol 2004;10:3332-3335.

13. Carlsson R, Dent J, Bolling-Sternevald E, et al. The usefulness of a structured questionnaire in the assessment of symptomatic gastroesophageal reflux disease. Scand J Gastroenterol 1998;33:1023-1029.

14. Shimoyama Y, Kusano M, Sugimoto S, et al. Diagnosis of gastroesophageal reflux disease using a new questionnaire. J Gastroenterol Hepatol 2005;20:643-647.

15. Kusano M, Shimoyama Y, Sugimoto S, et al. Devolopment and evaluation of FSSG: frequency scale for the symptoms of GERD. J Gastroenterol 2004;39:888-891.

16. Numans ME, de Wit NJ. Reflux symptoms in general practice: diagnostic evaluation of the Carlsson-Dent gasto-oesophageal reflux disease questionnaire. Aliment Pharmacol Ther 2003;17:1049-1055.

17. Hung CS, Lee CL, Yang JN, et al. Clinical application of Carlsson's questionnaire to predict erosive GERD among healthy Chinese. J Gastroenterol Hepatol 2005;20:1900-1905.

18. Chan K, Liu G, Miller L, et al. Lack of correlation between a self-administered subjective GERD questionnaire and pathological GERD diagnosed by 24-h esophageal monitoring. J Gastrointest Surg 2010; 14:427-436

19. Lundell LR, Dent J, Bennett JR, et al. Endoscopic assessment of esophagitis: clinical and functional correlates and further validation of the Los Angeles classification. Gut 1999;45:172-180.

20. Spechler SJ, Zeroogian JM, Antonioli DA, Wang HH, Goyal RK. Prevalence of metaplasia at the gastro-oesophageal junction. Lancet 1994;344:1533-1536.

21. Johnson LF, Demeester TR. Twenty-four-hour $\mathrm{pH}$ monitoring of distal esophagus. A quantitative measure of gastroesophageal reflux. Am J Gastroenterol 1974;62:325-332.

22. Scottish Intercollegiate Guidelines Network. Dyspepsia: a national clinical guideline. SIGN 2003. Available from: http://www.sign. ac.uk/pdf/sign68.pdf (accessed February 2011).

23. Talley NJ, Vakil N; the Practice Parameters Committee of the American College of Gastroenterology. Guidelines for the management of dyspepsia. Am J Gastroenterol 2005;100:2324-2337.

24. Talley NJ, Vakil NB, Moayyedi P. American gastroenterological association technical review on the evaluation of dyspepsia. Gastroenterology 2005;129:1756-1780.

25. Talley NJ, Lam SK, Goh KL, Fock KM. Management guidelines for uninvestigated and functional dyspepsia in the Asia-Pacific region: first asian pacific working party on functional dyspepsia. J Gastroenterol Hepatol 1998;13:335-353. 